\title{
openheart Long-term impact of baseline anaemia on clinical outcomes following percutaneous coronary intervention in stable angina
}

\author{
Line Davidsen (D , ${ }^{1}$ Kristian Hay Kragholm, ${ }^{1,2}$ Mette Aldahl, ${ }^{1}$ \\ Christoffer Polcwiartek, ${ }^{1,3}$ Christian Torp-Pedersen, ${ }^{1,4,5}$ Peter Soegaard, ${ }^{3}$ \\ Phillip Freeman ${ }^{3}$
}

\begin{abstract}
- Additional material is published online only. To view, please visit the journal online (http://dx.doi.org/10.1136/ openhrt-2020-001319)
\end{abstract}

To cite: Davidsen $\mathrm{L}$, Kragholm KH, Aldahl M, et al. Long-term impact of baseline anaemia on clinical outcomes following percutaneous coronary intervention in stable angina. Open Heart 2020;7:e001319. doi:10.1136/

openhrt-2020-001319

Received 7 May 2020

Revised 22 July 2020

Accepted 24 August 2020

Check for updates

(C) Author(s) (or their employer(s)) 2020. Re-use permitted under CC BY-NC. No commercial re-use. See rights and permissions. Published by BMJ.

For numbered affiliations see end of article.

Correspondence to

Dr Line Davidsen;

linedavidsen90@gmail.com

\section{ABSTRACT}

Background In patients with stable angina (SA), the clinical benefits of percutaneous coronary intervention (PCl) reside almost exclusively within the realm of symptomatic improvement rather than improvement in hard clinical endpoints. The benefits of $\mathrm{PCl}$ should always be balanced against its potential short-term and longterm risks. Common among these risks is the presence of anaemia and its interaction with poor clinical outcomes and increased morbidity; this study aims to elucidate the impact of anaemia on long-term clinical outcomes of this patient group.

Methods From Danish national registries, we identified patients with SA treated with $\mathrm{PCl}$ who had a haemoglobin measurement maximum of 90 days prior to $\mathrm{PCl}$ procedure. Anaemia was defined as haemoglobin $<130$ and $<120 \mathrm{~g} / \mathrm{L}$ in men and women, respectively. Follow-up was up to 3 years after $\mathrm{PCl}$, and Cox regression was used to estimate HRs with $95 \%$ Cls of hospitalisation due to bleeding, acute coronary syndrome (ACS) and all-cause mortality in patients with anaemia compared with patients without anaemia.

Results 0 f 2837 included patients, $14.6 \%$ had anaemia prior to $\mathrm{PCl}$. During follow-up, 93 patients (3.3\%) had a bleeding episode, which was higher in patients with anaemia (5.8\%) compared with patients without anaemia $(2.8 \%)$. A total of 213 patients $(7.5 \%)$ developed ACS, which was higher in patients with anaemia $(10.6 \%)$ compared with patients without anaemia (7.0\%). Furthermore, 185 patients $(6.5 \%)$ died, with a mortality rate of $18.1 \%$ in patients with anaemia compared with $4.5 \%$ in patients without anaemia. In multivariable analyses, anaemia was associated with a significantly increased risk of bleeding (HR 1.69; 95\% Cl 1.04 to 2.73; P 0.033), ACS (HR 1.47; 95\% Cl 1.04 to 2.10; P 0.031 ) and all-cause mortality (HR $2.41 ; 95 \% \mathrm{Cl} 1.73$ to $3.30 ; \mathrm{P}$ $<0.001$ ).

Conclusion Anaemia in patients with SA was significantly associated with bleeding, ACS and all-cause mortality following PCl.

\section{Key questions}

What is already known about this subject?

- Given evidence suggesting no survival benefit of percutaneous coronary intervention (PCl) in patients with stable angina, and $\mathrm{PCl}$ carries an additional risk of postprocedural bleeding.

- Baseline anaemia is recognised as an independent risk factor for bleeding and has previously been found as a predictor of in-hospital bleeding following PCl.

What does this study add?

- This study confirmed anaemia as an independent predictor of bleeding, acute coronary syndrome and mortality in the longer term, in patients with SA undergoing PCl.

How might this impact on clinical practice?

- Predicting a considerable bleeding risk prior to elective or ad hoc PCl by the means of a haemoglobin measurement is of great value and should modify our treatment strategies to more frequent use of medical therapy in these high-risk patients with relatively small reductions in haemoglobin.

\section{INTRODUCTION}

The optimal intervention strategy for stable angina (SA) remains controversial. ${ }^{12}$ In these patients, no survival benefit for percutaneous coronary intervention (PCI) has been shown when compared with medical therapy ${ }^{3-7}$ Previous studies have indicated better symptom relief and improved quality of life (QoL) for PCI-treated patients relative to medically treated patients. ${ }^{8}$ However, this may be a short-term benefit only, ${ }^{8}$ and PCI carries an additional risk of postprocedural bleeding, driven by the need for dual antiplatelet therapy or dual antiplatelet therapy together with oral anticoagulant therapy in an increasingly ageing population. ${ }^{9} 10$ 
Considering the postprocedural bleeding risk associated with PCI and that patients with SA often receive dual antiplatelet therapy and/or oral anticoagulant therapy, predicting a considerable bleeding risk prior to decision-making is of great value. Anaemia is already acknowledged as an independent risk factor for bleeding in the treatment of acute coronary syndrome (ACS) with PCI, ${ }^{11-16}$ thus assessing the haemoglobin status in patients with SA by means of risk assessment prior to PCI could be substantial. In addition, a haemoglobin level may also be associated with post-PCI ACS and mortality potentially driven by early cessation of dual antiplatelet therapy and blood/platelet transfusions.

Therefore, using nationwide register-based data on PCI procedures and haemoglobin levels from selected Danish regions, we studied bleeding requiring hospitalisation, ACS and all-cause mortality in relation to haemoglobin levels among patients with SA undergoing PCI in Denmark during 2004-2012.

\section{METHODS}

\section{Data source}

All residents in Denmark have a personal, unique and permanent civil registration number that enables individual linkage of personal data from all nationwide administrative registers. Data regarding sex and age were obtained from the Danish Civil Registration System. ${ }^{17}$ Survival status was gathered from the Danish Register of Causes of Death. ${ }^{18}$ Admission dates, procedure codes, operation codes, operation dates, hospital discharge dates and discharge diagnoses were collected from the Danish National Patient Register, which contains all hospital admissions in Denmark since 1978. ${ }^{19}$ Hospital discharge diagnosis is classified according to the WHO International Classification of Diseases (ICD). The 10th revision (ICD-10) has been applied from 1994 and onwards. The Danish National Prescription Registry includes all dispensed prescriptions from all Danish pharmacies since 1995 based on the Anatomical Therapeutic Chemical codes, and information about the pharmacotherapy of each patient was collected from here. ${ }^{20}$ As the healthcare system is state financed and the state partly reimburses drug costs, all Danish pharmacies are required by law to register all dispensed drug prescriptions, providing a valid and accurate register. From three regions in Denmark, with a source population of approximately 1.2 million inhabitants, blood test results were obtained from electronic registers of laboratory data.

\section{Study population}

The study population consists of Danish patients with a first-time stable coronary artery disease diagnosis between 2004 and 2012, either from a hospital or an ambulatory outpatient contact (online supplemental appendix 1). In addition, patients were required to have been treated with PCI following the stable coronary artery disease diagnosis (online supplemental appendix 2). Patients were excluded if they did not have a haemoglobin measurement on the day of PCI or maximum 90 days before. As information about the extent of antithrombotic therapy is of great importance for the study, baseline was set to be 7 days following the day of PCI, giving patients enough time to redeem a prescription for the medication after the procedure. Patients with an ACS diagnosis, PCI procedure or coronary artery bypass grafting procedure prior to the stable coronary artery disease diagnosis or PCI for ACS were excluded from the study population. Patients who were bleeding, suffering from ACS or died in the period between PCI until baseline $(+7$ days from PCI) were also excluded, as we demanded a 7-day period for patients to redeem prescriptions for relevant antithrombotic therapies post-PCI as mentioned above. We also excluded patients without a creatinine measurement on the day of PCI or maximum 90 days before. Thus, no patients in the study were missing baseline data. A flow chart is presented in online supplemental appendix 3.

\section{Comorbidities and concomitant pharmacotherapy}

Clinically relevant comorbidities and concomitant pharmacotherapy were identified and used as covariates in the analysis. Patients were considered having a specific comorbidity if the diagnosis was registered up to 10 years prior to the day of PCI. Comorbidities were identified from the Danish National Patient Register based on ICD-10 codes, and included diagnoses of heart failure, peripheral vascular disease, cerebral vascular disease, chronic obstructive pulmonary disease (COPD), peptic ulcer, hypertension, diabetes, cancer and atrial fibrillation. Diabetes and COPD are usually managed in primary care, and a diagnosis is therefore not necessarily registered. Thus, patients in the study were also considered to be suffering from diabetes or COPD if at least one prescription of antidiabetic medication or inhaled anticholinergic, beta adrenergic, or the combination of anticholinergic, beta adrenergic and/or corticosteroid inhalation medication had been dispensed at maximum of 180 days prior to PCI. Using ICD-10 codes, previous episodes of bleeding requiring hospitalisation were also identified up to 2 years prior to PCI. Renal status was evaluated by estimated glomerular filtration rate (eGFR). A creatinine measurement on the day of PCI or maximum 90 days before was used in the Chronic Kidney Disease Epidemiology Collaboration creatinine equation to calculate eGFR. Patients were considered having renal insufficiency with an eGFR $<30 \mathrm{~mL}$ / $\min / 1.73 \mathrm{~m}^{2}$. In addition, we identified patients treated with antithrombotic medication within $180+7$ days prior to baseline, and divided them into four groups (none, single, dual or triple therapy) depending on the number of different antithrombotic drugs they were treated with. The antithrombotic medication included in the study was acetylsalicylic acid, heparin, non-vitamin K antagonist oral anticoagulants, platelet inhibitors and vitamin $\mathrm{K}$ antagonist (online supplemental appendices 1 and 4). 


\section{Definition of anaemia}

Baseline demographics and clinical characteristics were compared by dividing all patients into two groups based on haemoglobin levels. Patients with a blood haemoglobin concentration less than $8.1 \mathrm{mmol} / \mathrm{L}$ (corresponding to $130 \mathrm{~g} / \mathrm{L}$ ) in men and less than $7.5 \mathrm{mmol} / \mathrm{L}$ (corresponding to $120 \mathrm{~g} / \mathrm{L}$ ) in women were classified as having anaemia. Both groups were according to the cutoff values defined by the WHO. ${ }^{21}$ The final study population combined the classification of men and women as anaemic or non-anaemic.

\section{Outcomes}

The primary outcome of interest was hospitalisation due to cerebral, gastrointestinal or urogenital bleeding in a follow-up period of 3 years. Secondary outcomes were ACS, including fatal ACS, and all-cause mortality. Bleeding and ACS were identified from the Danish National Patient Register based on ICD-10 codes (online supplemental appendix 1). The study population was followed from baseline until bleeding, ACS, or mortality, or until patients were censored 3 years after baseline, depending on whichever came first.

\section{Statistical analysis}

Categorical variables were presented as counts and percentages, and continuous variables as median with 25-75 percentiles. Cumulative incidence of bleeding and ACS was estimated using the Aalen-Johansen estimator, treating mortality as a competing risk. Survival was depicted using Kaplan-Meier estimates. A Cox proportional hazards regression model was used to determine the association between anaemia and bleeding, ACS and all-cause mortality. From these models, HRs with 95\% CIs were reported. A two-sided p value below 0.05 was considered statistically significant. The proportional hazard assumption was tested for all regression models and was fulfilled. In addition, the assumption of linearity was tested for age, and because of lack of linearity, age was included in the regression model as four groups (<60 years, 60-69 years, 70-79 years, >79 years). We tested for interactions using a log likelihood ratio test. A two-sided p value below 0.01 was considered statistically significant, and no interactions were found. In the multivariable analysis of bleeding we adjusted for sex, age groups, renal insufficiency, peptic ulcer, cancer, hypertension and antithrombotic therapy. The adjusted analysis of all-cause mortality included the following covariates: sex, age groups, renal insufficiency, heart failure, peripheral vascular disease, cerebral vascular disease, COPD, hypertension, diabetes, cancer, peptic ulcer and antithrombotic therapy. Multivariable analysis of ACS included the same covariates as the analysis of all-cause mortality except peptic ulcer. All data management and analyses were performed using SAS (V.9.4, SAS Institute) and R statistics V.3.5.0 (R Development Core Team). ${ }^{22}$

\begin{tabular}{|c|c|c|c|}
\hline & $\begin{array}{l}\text { No anaemia } \\
(n=2423)\end{array}$ & $\begin{array}{l}\text { Anaemia } \\
(n=414)\end{array}$ & $P$ value \\
\hline \multicolumn{4}{|l|}{ Haemoglobin } \\
\hline Mean (SD) & $8.93(0.66)$ & $7.26(0.66)$ & $<0.0001$ \\
\hline \multicolumn{4}{|l|}{ Sex } \\
\hline Female & $684(28.2 \%)$ & $126(30.4 \%)$ & \\
\hline Male & $1739(71.8 \%)$ & $288(69.6 \%)$ & 0.3901 \\
\hline \multicolumn{4}{|l|}{ Age } \\
\hline Median (p25-p75) & $64.9(58.3,71.8)$ & $70.8(62.3,78.0)$ & $<0.0001$ \\
\hline Heart failure & 225 (9.3\%) & $95(22.9 \%)$ & $<0.0001$ \\
\hline $\begin{array}{l}\text { Peripheral vascular } \\
\text { disease }\end{array}$ & 178 (7.3\%) & $56(13.5 \%)$ & $<0.0001$ \\
\hline $\begin{array}{l}\text { Cerebral vascular } \\
\text { disease }\end{array}$ & $167(6.9 \%)$ & $51(12.3 \%)$ & 0.0002 \\
\hline $\begin{array}{l}\text { Chronic obstructive } \\
\text { pulmonary disease }\end{array}$ & $249(10.3 \%)$ & $68(16.4 \%)$ & 0.0003 \\
\hline Peptic ulcer & $126(5.2 \%)$ & $34(8.2 \%)$ & 0.0193 \\
\hline Cancer & $182(7.5 \%)$ & $46(11.1 \%)$ & 0.0168 \\
\hline Atrial fibrillation & 187 (7.7\%) & $57(13.8 \%)$ & $<0.0001$ \\
\hline Hypertension & $915(37.8 \%)$ & $202(48.8 \%)$ & 0.0011 \\
\hline Diabetes & $371(15.3 \%)$ & $111(26.8 \%)$ & $<0.0001$ \\
\hline Renal insufficiency & $21(0.9 \%)$ & $35(8.5 \%)$ & $<0.0001$ \\
\hline Previous bleeding & $8(0.3 \%)$ & $10(2.4 \%)$ & $<0.0001$ \\
\hline \multicolumn{4}{|l|}{$\begin{array}{l}\text { Antithrombotic } \\
\text { therapy }\end{array}$} \\
\hline None & $92(3.8 \%)$ & $26(6.3 \%)$ & \\
\hline Single & $546(22.5 \%)$ & $112(27.1 \%)$ & \\
\hline Dual & $1686(69.6 \%)$ & 247 (59.7\%) & \\
\hline Triple & $99(4.1 \%)$ & $29(7.0 \%)$ & 0.0002 \\
\hline
\end{tabular}

Data are presented as number of patients (column percentage) in all parameters except for age and haemoglobin. Data are in the age parameter presented as median and the 25th and 75th percentiles, and in the haemoglobin parameter as mean and SD.

\section{RESULTS}

\section{Baseline characteristics}

The study population consisted of 2837 patients with SA treated with PCI who had an available haemoglobin measurement (online supplemental appendix 3). Baseline characteristics of these patients were mainly comparable to baseline characteristics of the excluded patients without a haemoglobin measurement (online supplemental appendix 6).

The baseline characteristics of the study population are presented in table 1. Of the 2837 patients, 414 (14.6\%) were anaemic. The distribution of haemoglobin levels in the population is presented in online supplemental appendix 7 . The overall population was characterised by advanced age (median: 65.5 ; $25-75$ percentiles: $58.7-$ 72.7) and the majority of patients were male $(71.4 \%)$. Compared with patients without anaemia, patients with anaemia were older (no anaemia, median: 64.9; anaemia, median: 70.8) and had a higher comorbidity 


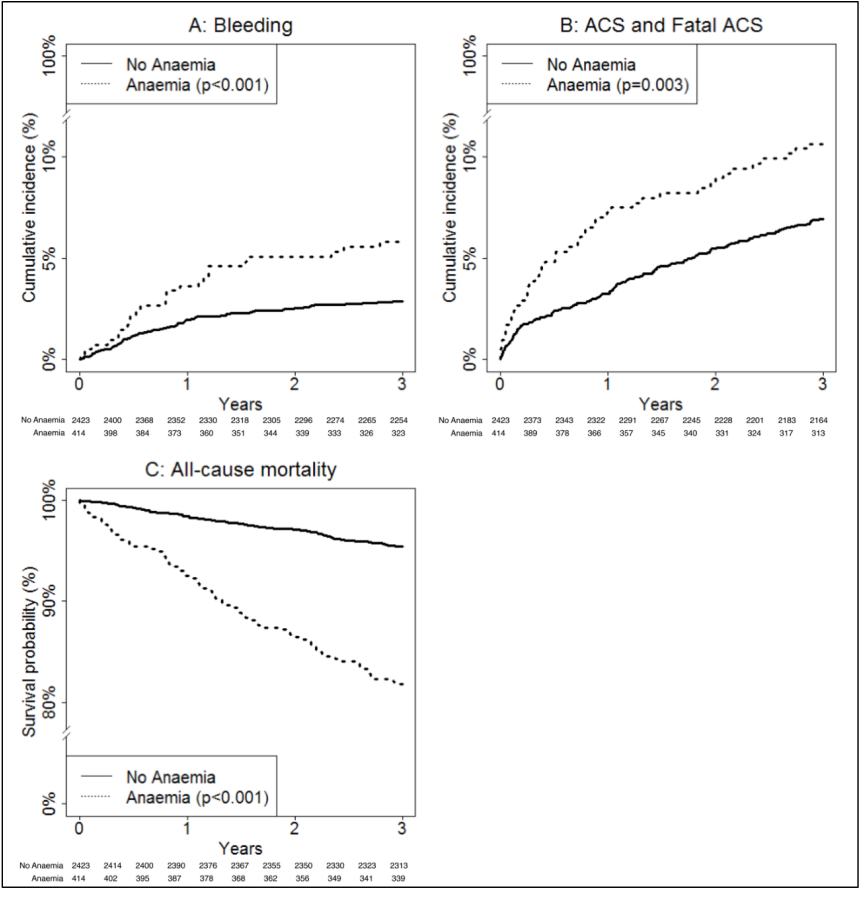

Figure $1 P$ values indicate the difference between patients with and without anaemia and are based on an unadjusted Cox regression model. Numbers at risk are presented below the figures. (A) Cumulative incidence of bleeding during the 3 years of follow-up, among patients with and without anaemia. (B) Cumulative incidence of acute coronary syndrome (ACS) and fatal ACS during the 3 years of followup, among patients with and without anaemia. (C) KaplanMeier chart of probability of survival during the 3 years of follow-up, among patients with and without anaemia.

burden. The baseline use of antithrombotic agents was as follows: $118(4.2 \%)$ patients were not treated with antithrombotic medicine, $658(23.2 \%)$ patients received

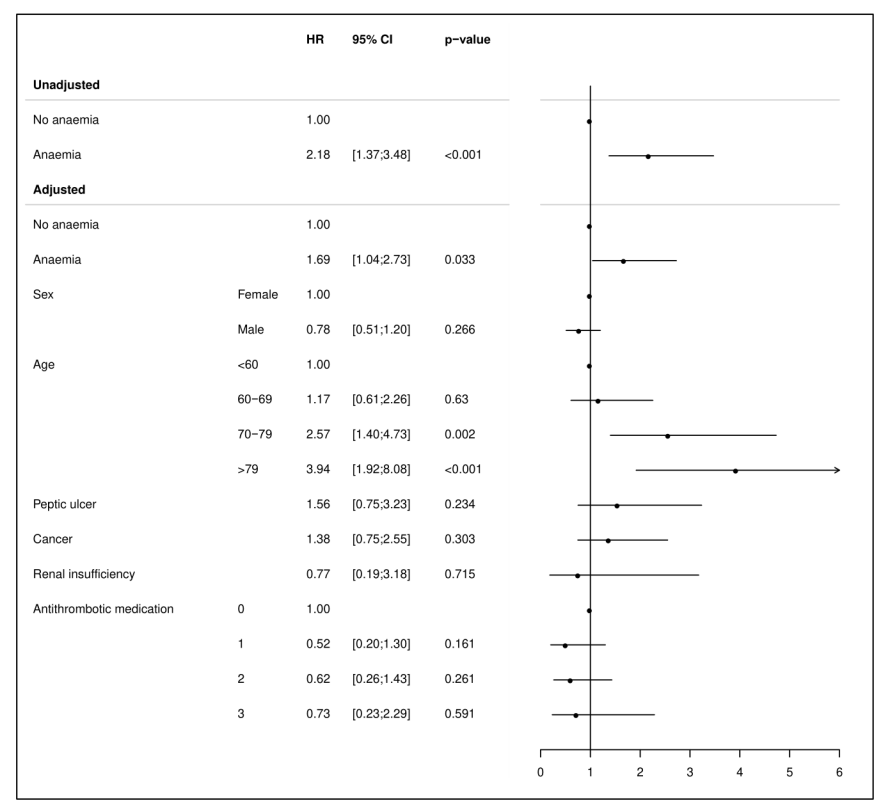

Figure 2 HR for 3-year bleeding associated with anaemia in patients with stable angina treated with percutaneous coronary intervention. single antithrombotic therapy, $1933(68.1 \%)$ patients received dual antithrombotic therapy and 128 (4.5\%) patients received triple antithrombotic therapy. The components of dual antithrombotic therapy were in 1870 $(96.7 \%)$ patients treated with two antiplatelet drugs, the remaining $63(3.3 \%)$ patients were treated with one antiplatelet and one anticoagulant drug.

\section{Anaemia and risk of bleeding}

In the final study population of 2837 patients, a total of $93(3.3 \%)$ had a bleeding requiring hospitalisation within 3 years from baseline. The mean follow-up was 1035 days (2.8 years). Patients with anaemia had a significantly higher proportion of bleeding events (5.8\%; 95\% CI $3.55 \%$ to $8.07 \%$ ) compared with patients without anaemia $(2.9 \%$; $95 \%$ CI $2.19 \%$ to $3.52 \%)$. The 3 -year cumulative incidence is illustrated in figure 1A. The majority of bleeding events were gastrointestinal bleeding $(87.1 \%)$, while the remaining were cerebral bleeding $(8.6 \%)$ and urogenital bleeding $(4.3 \%)$. The results of the unadjusted and adjusted Cox regression analyses are shown in figure 2. The unadjusted analysis showed a twofold significant increased risk of bleeding in patients with anaemia compared with patients without anaemia (HR 2.18; 95\% CI 1.37 to 3.48; $\mathrm{P}<0.001$ ). After multivariable adjustment, the risk of bleeding remained significantly increased in patients with anaemia (HR 1.69; $95 \%$ CI 1.04 to 2.73 ; P 0.033 ). Age was the only covariate with a significant impact on bleeding, where an increased risk of bleeding was observed in patients aged 70 years and above.

\section{Anaemia and risk of ACS and fatal ACS}

In the follow-up period of 3 years from baseline, 213 $(7.5 \%)$ patients had ACS including fatal ACS. The mean follow-up was 1013 days (2.7 years). Throughout the 3 years, patients with anaemia had a significantly higher cumulative incidence at $10.6 \%$ (95\% CI $7.68 \%$ to $13.63 \%$ ) compared with patients without anaemia $(7.0 \%$; $95 \%$ CI $5.94 \%$ to $7.97 \%$ ), as illustrated in figure 1B. In figure 3 , the results of the unadjusted and adjusted Cox regression analyses are shown. Patients with anaemia compared with patients without anaemia had a significantly increased risk of ACS (HR 1.65; 95\% CI 1.18 to 2.30; P 0.003). When adjusting for multiple covariates, the risk of ACS remained significantly increased in patients with anaemia (HR 1.47; 95\% CI 1.04 to 2.10; P 0.031). Diabetes was the only confounder with a significant impact on ACS.

\section{Anaemia and risk of all-cause mortality}

A total of 185 (6.5\%) patients died within 3years from baseline. The mean follow-up was 1057 days (2.9 years). The main cause of death was cardiovascular disease $(46.5 \%)$ followed by cancer (25.9\%) (online supplemental appendix 5). Patients with anaemia had a significantly higher mortality rate at $18.1 \%$ (95\% CI $14.4 \%$ to $21.9 \%$ ) compared with patients without anaemia $(4.5 \%$; $95 \%$ CI $3.7 \%$ to $5.4 \%)$. In figure 1C, the Kaplan-Meier survival probability is illustrated 


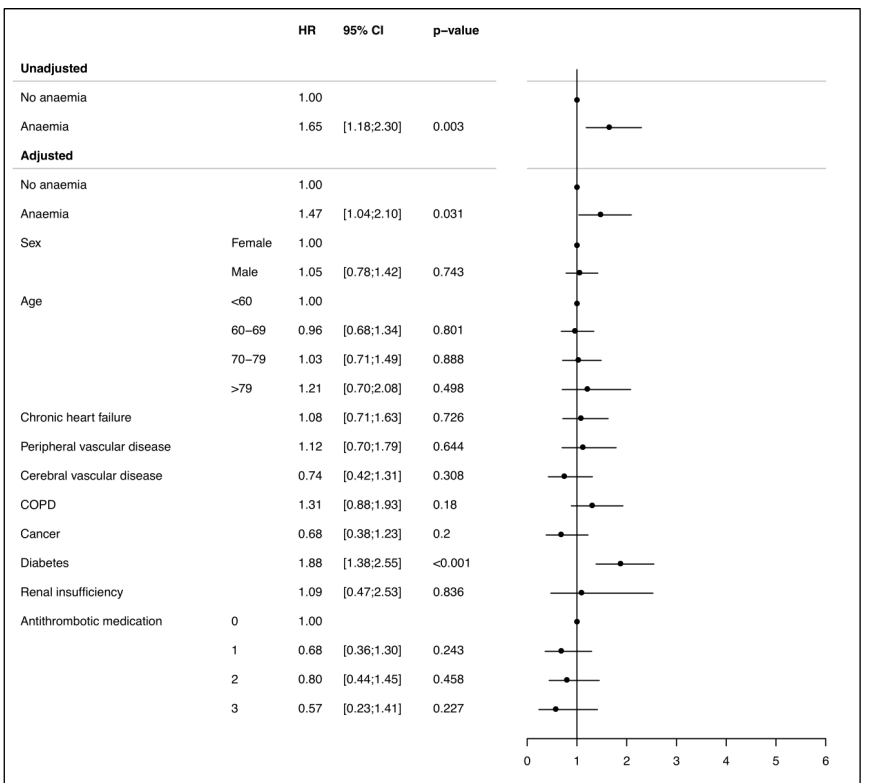

Figure 3 HR for 3-year acute coronary syndrome and fatal acute coronary syndrome associated with anaemia in patients with stable angina treated with percutaneous coronary intervention. COPD, chronic obstructive pulmonary disease.

with diverging curves throughout all 3years of follow-up, showing a distinct decrease in survival probability in patients with anaemia. The results of the unadjusted and adjusted Cox regression analyses are shown in figure 4 . The unadjusted analysis revealed a fourfold significant increased risk of all-cause mortality in patients with anaemia compared with patients without anaemia (HR 4.33; 95\% CI 3.23 to 5.81; P $<0.001)$. This remained significant when adjusting for covariates, with a more than twofold increased risk of all-cause

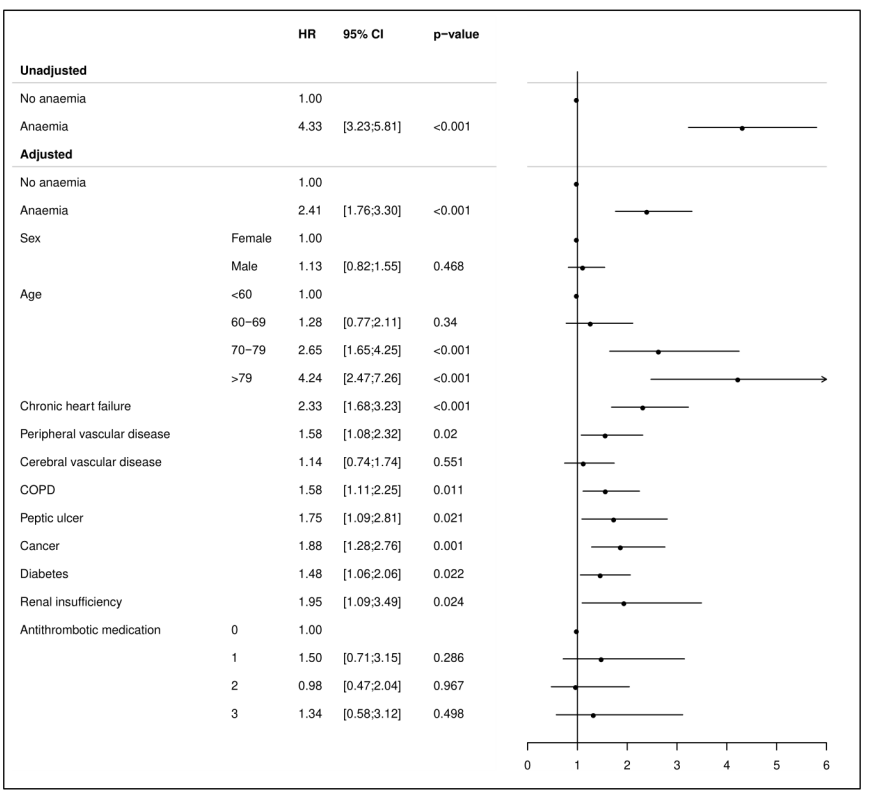

Figure 4 HR for 3-year all-cause mortality associated with anaemia in patients with stable angina treated with percutaneous coronary intervention. COPD, chronic obstructive pulmonary disease. mortality in patients with anaemia (HR 2.41; 95\% CI 1.76 to 3.30; $\mathrm{P}<0.001)$. Covariates with a significant impact on allcause mortality were age, renal insufficiency, COPD, heart failure, cancer, peripheral vascular disease, diabetes and peptic ulcer.

\section{Additional analyses}

To test the strength of the results, additional analyses were performed. Analysis on a subgroup including only patients with anaemia was performed $(n=414)$. First, the association between bleeding requiring hospitalisation and increasing age, renal insufficiency, peptic ulcer and cancer was determined (see online supplemental appendix 8). Second, the association between ACS and increasing age, renal insufficiency, chronic heart failure, peripheral vascular disease, cerebral vascular disease, diabetes and cancer was determined (see online supplemental appendix 9). Third, the association between allcause mortality and increasing age, renal insufficiency, chronic heart failure, peripheral vascular disease, cerebral vascular disease, COPD, diabetes and cancer was determined (see online supplemental appendix 10).

\section{DISCUSSION}

In this Danish cohort study of patients with SA undergoing PCI, we investigated the association between anaemia and bleeding, ACS and mortality in a long-term follow-up period of 3 years. Anaemia was present in $14.6 \%$ of the entire study population and associated with a significant increased risk of bleeding. Furthermore, an increased risk of ACS in patients with anaemia was present, and anaemia was highly associated with all-cause mortality. Thus, along with other studies, our results confirm that anaemia was an independent predictor of bleeding, ACS and mortality in patients with SA undergoing PCI, even after adjusting for covariates.

Identification of factors associated with significant complications in the treatment of SA is clearly important in a disease that has a relatively low frequency of major cardiac adverse events, and in which invasive treatment with PCI offers just symptomatic treatment in the majority of cases. ${ }^{23}$ For some patients, an invasive treatment may even cause more harm than benefit. We have determined that bleeding complications requiring hospitalisation following treatment with PCI in these patients were significantly associated with anaemia. This association has previously only been found in a study investigating in-hospital bleeding. ${ }^{24}$ Hence, this study is, to our knowledge, the first to present anaemia as a predictor of bleeding following elective PCI in the longer term. Our results are also consistent with findings of studies examining anaemia as a predictor of bleeding in all patients undergoing PCI, including patients with ACS. ${ }^{1-16}$ We measured bleeding events requiring hospitalisation, meaning that bleeding events were notable and could be considered major. In addition, we also revealed a significant association between anaemia and an increased risk of ACS and all-cause mortality, which is consistent with 
previous studies. ${ }^{25-27}$ The relationship between bleeding, ACS and death is complicated with many factors. Potential mechanisms may include an increased rate of ACS after premature cessation of antiplatelet therapy, but we can only speculate on actual mechanisms. Previous studies have already found bleeding to be associated with increased risk of mortality following PCI, ${ }^{9} 1028$ which might be related to recurrent myocardial infarction and complications following recurrent myocardial infarction, such as heart failure and arrhythmias. Thus, it is plausible that bleeding requiring hospitalisation may increase the risk of further complications following PCI. Predicting a considerable bleeding risk prior to elective or ad hoc PCI by the means of a haemoglobin measurement is of great value and should modify our treatment strategies to more frequent use of medical therapy in these high-risk patients with relatively small reductions in haemoglobin.

In this study population, patients from Danish national registers were included. Although patients missing a haemoglobin measurement were excluded, characteristics of patients with and without a haemoglobin measurement were mainly comparable, increasing the generalisability of the study to a nationwide level. In addition, only few other exclusion criteria were present, reflecting the realworld population of patients with SA undergoing PCI. In contrast, the majority of previous randomised controlled trials tend to exclude older patients and patients with severe comorbidities, creating a selective population with considerable differences from this real-world population. Only one previous study has investigated conservative treatment versus PCI in patients aged 75 or older. ${ }^{29}$ Consistent with other trials, the benefits of PCI found in the study of the elderly were limited to a relief in symptoms and improvement in QoL. ${ }^{3-6} 82930$ The Objective Randomised Blinded Investigation with optimal medical Therapy of Angioplasty in stable angina (ORBITA) trial compared for the first time PCI with placebo (sham procedure) in patients with $\mathrm{SA}$, and powered to detect a relatively modest $16.6 \mathrm{~s}$ change in exercise time. ORBITA found no significant difference in exercise time. ${ }^{7}$ Despite its several limitations, ORBITA raises questions about the efficacy of PCI over medical therapy for symptom control in patients with SA. Although this finding was limited by a short follow-up time of 6 weeks, the significant benefits of medical therapy that was initiated in both groups were likely responsible for the non-significant difference in exercise time. These studies highlight that in an elderly population with baseline anaemia we do have viable alternatives to invasive treatments.

Compared with patients without anaemia, we found that patients with anaemia were older and had a greater proportion of comorbidities, which is similar to results from previous studies. ${ }^{112} 1624-2631$ However, after adjustment, anaemia continued having a significant association with all endpoints. Most randomised trials in SA investigating the effect of PCI have historically excluded older patients with more frequent comorbidities, highlighting the limited knowledge we have for the optimal management strategy for these high-risk patients with anaemia reflected in our data. Based on the results of this study, it is undeniable that patients with anaemia and SA deserve more attention, as they are at a higher risk of adverse outcomes when treated with PCI, and that PCI procedure in these patients may be more harmful than beneficial.

\section{Study limitations}

Some limitations should be considered. Due to an observational design, our results should be interpreted only as associative and not causal relations. Unmeasured confounders could still be present in our study, despite using Danish registers to include information on different potential confounders for adjustment purposes. Details from PCI procedures were not available, including information about the degree of stenosis. No data on how long patients were treated with antithombotic medicine were available. A control group with patients being conservatively treated with medicine alone was also not available, as it was very difficult to accurately define patients with SA not treated with revascularisation from the available registry sources. Additionally, it is unknown whether anaemia was persistent or transient throughout follow-up, as haemoglobin levels were only measured before baseline. Furthermore, we were not able to identify patients receiving blood transfusion prior to PCI, since data of blood transfusion were not available. Due to small population size and thus relatively few bleeding events, we were unable to comment on the aetiology of anaemia, and whether different aetiologies had a different effect on the outcomes following PCI. No quantification of bleeding severity was available, therefore, we chose to analyse clinically significant bleeding events that required hospitalisation. Finally, most of the population in Denmark consists of a white Caucasian population, and generalisation of these data to other racial and ethnic groups should be cautioned.

\section{Clinical implications}

Although no comparison group of medically treated SA was available, we demonstrated a higher bleeding risk among patients with anaemia undergoing PCI. Given evidence suggesting no benefit of PCI for $\mathrm{SA}^{78}$ on hard endpoints and only limited benefit for symptom relief, our study suggests that in patients with greater risk for harm than benefit, that is, patients who are older and with anaemia, medical therapy should be seen as a viable alternative. These observational data warrant prospective and randomised research to further elucidate this issue.

\section{CONCLUSION}

Anaemia in patients with SA was significantly associated with bleeding, ACS and all-cause mortality following PCI.

Author affiliations

${ }^{1}$ Department of Clinical Epidemiology, Aalborg Universitetshospital, Aalborg, Denmark 
${ }^{2}$ Department of Cardiology, Regionshospital Nordjylland, Hjørring, Nordjylland, Denmark

${ }^{3}$ Department of Cardiology and Clinical Medicine, Aalborg University Hospital, Aalborg, Nordjylland, Denmark

${ }^{4}$ Department of Health, Science and Technology, Aalborg Universitet Det Sundhedsvidenskabelige Fakultet, Aalborg, Denmark

${ }^{5}$ Department of Cardiology, Nordsjællands Hospital, Hillerød, Denmark

Contributors Conception and design of the work: LD, PMF, PS, KHK and CTP. Data collection: LD, MA and KHK. Data analysis and interpretation: LD, MA, KHK, PMF and PS. Drafting the article: LD, KHK and PMF. Critical revision of the article: PMF, PS, KHK, CP and CTP. Final approval of the version to be published: LD, MA, PMF, PS, KHK, CP and CTP.

Funding The authors have not declared a specific grant for this research from any funding agency in the public, commercial or not-for-profit sectors.

Competing interests KHK has received personal fees and/or grants from Novartis and the Laerdal Foundation. CP reports receiving speaker fees from Lundbeck Pharma, and research grants from the Danish Heart Foundation and the Eva and Henry Frænkel Memorial Foundation. PMF has received speaker fees and grants from AstraZeneca, Edwards Lifesciences, Abbott and Roche Diagnostics. PS reports being consultant at Biotronik.

Patient consent for publication Not required.

Ethics approval Register studies do not need ethical approval in Denmark. The study was approved by the data responsible unit in the capital region of Denmark (P-2019-404)

Provenance and peer review Not commissioned; externally peer reviewed.

Data availability statement No data are available. All data originate from Danish national registries, and are not available to the public.

Open access This is an open access article distributed in accordance with the Creative Commons Attribution Non Commercial (CC BY-NC 4.0) license, which permits others to distribute, remix, adapt, build upon this work non-commercially, and license their derivative works on different terms, provided the original work is properly cited, appropriate credit is given, any changes made indicated, and the use is non-commercial. See: http://creativecommons.org/licenses/by-nc/4.0/.

ORCID iD

Line Davidsen http://orcid.org/0000-0002-1120-8144

\section{REFERENCES}

1 Knuuti J, Wijns W, Saraste A, Achenbach S, et al. 2019 ESC guidelines for the diagnosis and management of chronic coronary syndromes. Eur Heart J 2020;41:407-77.

2 Neumann F-J, Sousa-Uva M, Ahlsson A, et al. 2018 ESC/ EACTS guidelines on myocardial revascularization. Eur Heart $J$ 2019;40:87-165.

3 Khan SU, Singh M, Lone AN, et al. Meta-analysis of long-term outcomes of percutaneous coronary intervention versus medical therapy in stable coronary artery disease. Eur J Prev Cardiol 2019;26:1-4.

4 Stergiopoulos K, Brown DL. Initial coronary stent implantation with medical therapy vs medical therapy alone for stable coronary artery disease: meta-analysis of randomized controlled trials. Arch Intern Med 2012;172:312-9.

5 Wijeysundera HC, Nallamothu BK, Krumholz HM, et al. Metaanalysis: effects of percutaneous coronary intervention versus medical therapy on angina relief. Ann Intern Med 2010;152:370-9.

6 Boden WE, O'Rourke RA, Teo KK, et al. Optimal medical therapy with or without $\mathrm{PCl}$ for stable coronary disease. $N$ Engl J Med 2007;356:1503-16.

7 Al-Lamee R, Thompson D, Dehbi H-M, et al. Percutaneous coronary intervention in stable angina (ORBITA): a double-blind, randomised controlled trial. Lancet 2018;391:31-40.

8 Weintraub WS, Spertus JA, Kolm P, et al. Effect of $\mathrm{PCI}$ on quality of life in patients with stable coronary disease. $N$ Engl J Med 2008;359:677-87.
9 Valle JA, Shetterly S, Maddox TM, et al. Postdischarge bleeding after percutaneous coronary intervention and subsequent mortality and myocardial infarction: insights from the $\mathrm{HMO}$ research network-stent registry. Circ Cardiovasc Interv 2016;9:1-9.

10 Ndrepepa G, Guerra E, Schulz S, et al. Weight of the bleeding impact on early and late mortality after percutaneous coronary intervention. J Thromb Thrombolysis 2015;39:35-42.

11 Kwok CS, Tiong D, Pradhan A, et al. Meta-Analysis of the prognostic impact of anemia in patients undergoing percutaneous coronary intervention. Am J Cardiol 2016;118:610-20.

12 Ali ZA, Poludasu S, Qureshi YH, et al. Impact of major bleeding on long-term mortality in anemic versus nonanemic patients undergoing percutaneous coronary intervention using bivalirudin. Am J Cardiol 2014;113:1481-6.

13 Pilgrim T, Rothenbühler M, Kalesan B, et al. Additive effect of anemia and renal impairment on long-term outcome after percutaneous coronary intervention. PLoS One 2014;9:e114846.

14 Feit F, Voeltz MD, Attubato MJ, et al. Predictors and impact of major hemorrhage on mortality following percutaneous coronary intervention from the REPLACE-2 trial. Am J Cardiol 2007:100:1364-9.

15 Nikolsky E, Mehran R, Dangas G, et al. Development and validation of a prognostic risk score for major bleeding in patients undergoing percutaneous coronary intervention via the femoral approach. Eur Heart J 2007;28:1936-45

16 Voeltz MD, Patel AD, Feit F, et al. Effect of anemia on hemorrhagic complications and mortality following percutaneous coronary intervention. Am J Cardiol 2007;99:1513-7.

17 Pedersen CB. The Danish civil registration system. Scand J Public Health 2011;39:22-5.

18 Helweg-Larsen K. The Danish register of causes of death. Scand J Public Health 2011;39:26-9.

19 Lynge E, Sandegaard JL, Rebolj M. The Danish national patient register. Scand J Public Health 2011;39:30-3.

20 Kildemoes HW, Sørensen HT, Hallas J. The Danish national prescription registry. Scand J Public Health 2011;39:38-41.

21 WHO. Haemoglobin concentrations for the diagnosis of anaemia and assessment of severity. Vitam Miner Nutr Inf Syst World Heal Organ 2011.

22 R Core Team. R: a language and environment for statistical computing, 2018.

23 Maron DJ, Hochman JS, Reynolds HR, et al. Initial invasive or conservative strategy for stable coronary disease. N Engl J Med 2020;382:1395-407.

24 Numasawa Y, Ueda I, Sawano M, et al. Relation of baseline hemoglobin level to in-hospital outcomes in patients who undergo percutaneous coronary intervention (from a Japanese multicenter registry). Am J Cardiol 2018;121:695-702.

25 Hosseini SK, Ansari MJA, Lotfi Tokaldany M, et al. Association between preprocedural hemoglobin level and 1-year outcome of elective percutaneous coronary intervention. J Cardiovasc Med 2014;15:331-5

26 Kitai Y, Ozasa N, Morimoto T, et al. Prognostic implications of anemia with or without chronic kidney disease in patients undergoing elective percutaneous coronary intervention. Int J Cardiol 2013:168:5221-8.

27 Catakoglu AB, Aytekin S, Sener M, et al. Impact of anemia on nonfatal coronary events after percutaneous coronary interventions. Heart Vessels 2007;22:383-8.

28 Kazi DS, Leong TK, Chang TI, et al. Association of spontaneous bleeding and myocardial infarction with long-term mortality after percutaneous coronary intervention. J Am Coll Cardiol 2015;65:1411-20.

29 TIME Investigators. Trial of invasive versus medical therapy in elderly patients with chronic symptomatic coronary-artery disease (time): a randomised trial. Lancet 2001;358:951-7.

30 Katritsis DG, loannidis JPA. Percutaneous coronary intervention versus conservative therapy in nonacute coronary artery disease: a meta-analysis. Circulation 2005;111:2906-12.

31 Pilgrim T, Vetterli F, Kalesan B, et al. The impact of anemia on longterm clinical outcome in patients undergoing revascularization with the unrestricted use of drug-eluting stents. Circ Cardiovasc Interv 2012;5:202-10. 